\title{
Numerical modeling negative corona under the influence of applied voltage variation
}

\author{
Hala Alnaemi ${ }^{1 *}$, Qais Algwari ${ }^{2}$ \\ ${ }^{1 *}$ Department of Physics, College of Education for Pure Science, Mosul University, Mosul, Iraq \\ ${ }^{2}$ Electronic Department, College of Electronics Engineering, Nineveh University, Mosul, Iraq \\ E-mail: ${ }^{1 *}$ halalneami74@gmail.com, ${ }^{2}$ qais.thanon@gmail.com
}

(Received October 27, 2020; Accepted December 22, 2020; Available online June 01, 2021)

DOI: 10.33899/edusj.2020.128762.1120, (C) 2021, College of Education for Pure Science, University of Mosul.

This is an open access article under the CC BY 4.0 license (http://creativecommons.org/licenses/by/4.0/).

\begin{abstract}
In high-voltage gaseous insulation systems, the corona discharge is important because it can lead to drop of the insulating qualities of the gas in addition to the production of harm by-products. The influence of the applied voltage variation on the negative corona characteristics in a coaxial electrode geometry has been investigated based on one-dimensional dynamic model of corona discharge includes one-dimensional continuity equations and Poisson equation. The one-dimensional fluid model of corona discharge is solved by finite difference flux correction method (FD-FCT). The calculations were performed on oxygen gas under the atmospheric pressure using COMSOL multiphysics software. The corona discharge parameters are simulated under different voltage values $4 \mathrm{KV}, 6 \mathrm{KV}, 7 \mathrm{KV}, 8 \mathrm{KV}$ and $10 \mathrm{KV}$, respectively. The effect of the applied voltage on the spatial distribution of main charged species and electron as well as the ozone was considered. The results show that as the negative applied voltage on the cathode increased the total current density also increase while the electron density decrease. The ozone density do not much affected by the increasing the applied voltage.
\end{abstract}

Keywords: Negative Corona Discharge, Ozone Density, Negative applied Voltage

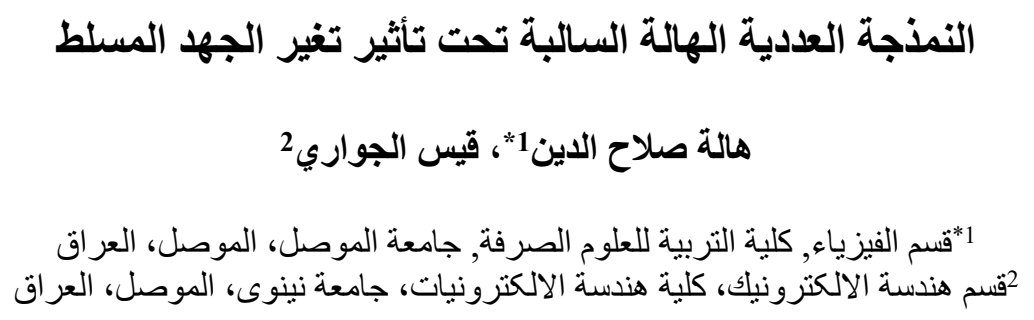

الخلاصة

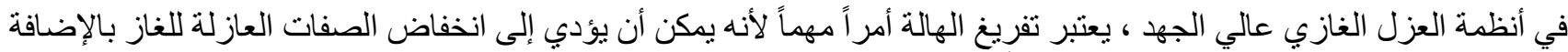

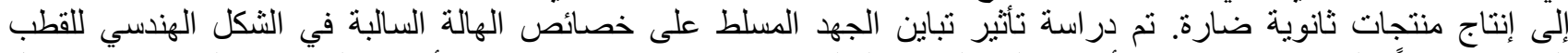

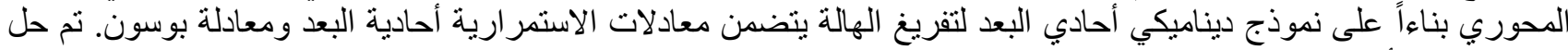

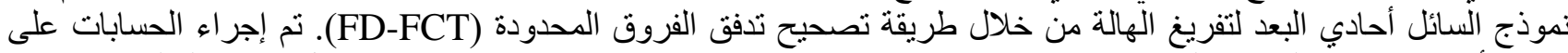
غاز الأكسجين تحت الضغط الجوي باستخدام برنامج COMSOL multiphysics. تم محاكاة عو امل تفريغ الهالة تحت جهد 
مختلف عند 4 كيلو فولت , 6 كيلو فولت , 7 كيلو فولت , 8 كيلو فولت , 10 كيلو فولت )على التوالتي. تم النظر في تأثير الجهد

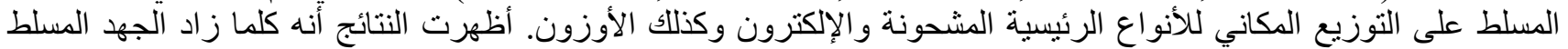

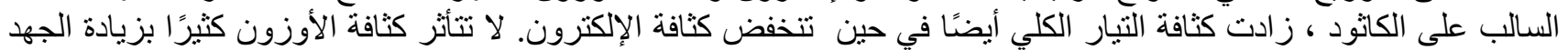
المسلط.

الكلمات المفتاحية: تفريغ الهالة السالبة, كثافة الاوزون, الجهد المسلط السالب .

المقدمة

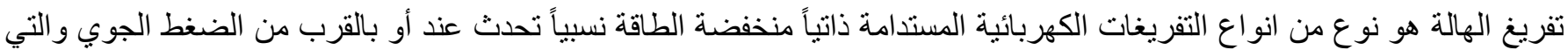

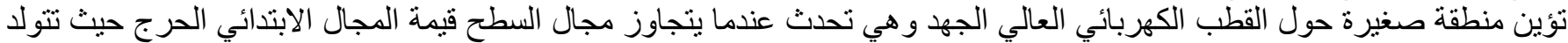

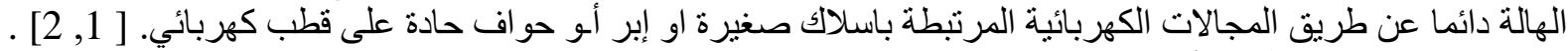

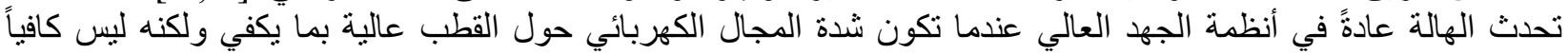

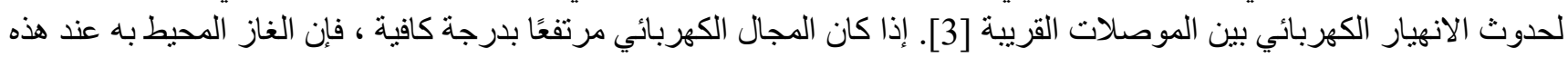

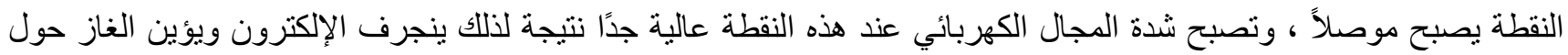

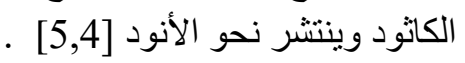

تبدأ عملية الهالة بجهذ يسمى الجهد الابتدائي و التي تعتمد على العديد من العو امل مثل القطبية وشكل القطب الكهربائي وضغط الغاز. يمكن أن يحدث الانهيار عند جهد أعلى من الجهد الابتدائي للهالة.

اعتماداً على قطبية الجهد الكهربائي المسلط على القطب الكهربائي المدبب ، يمكن تصنيف تفريغ الهالة على أنه موجباً او سالباً

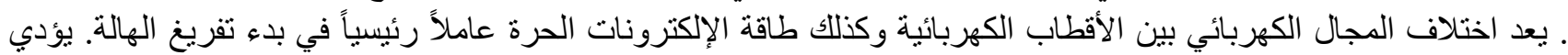

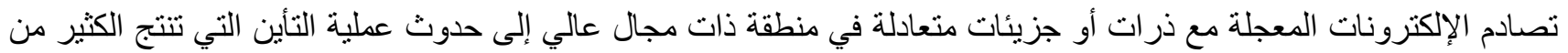

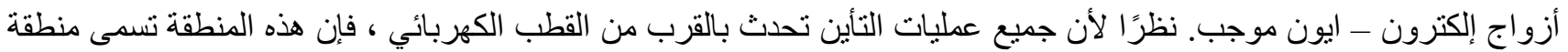

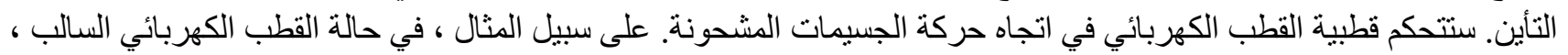

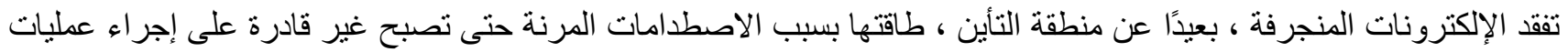

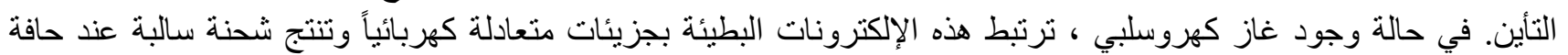

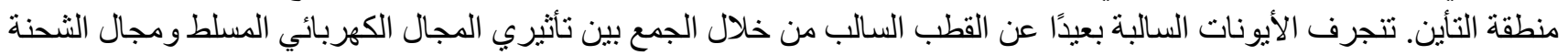

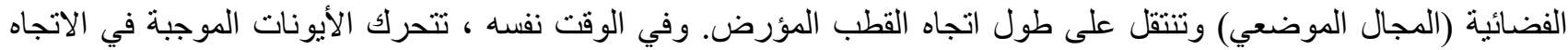

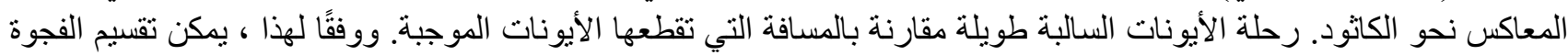

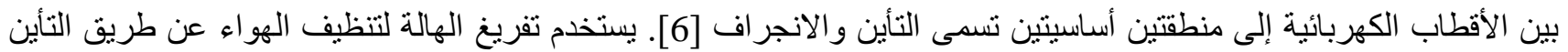

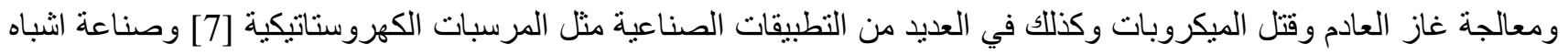

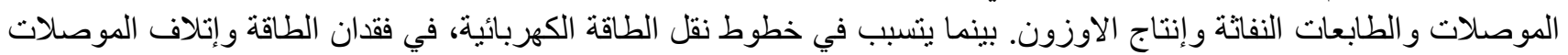

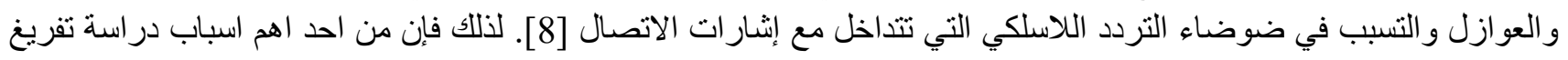

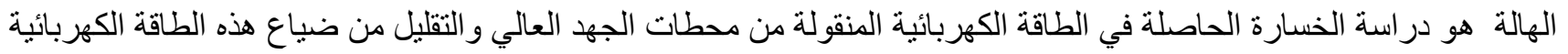

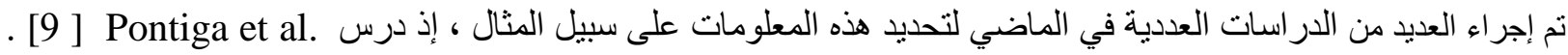

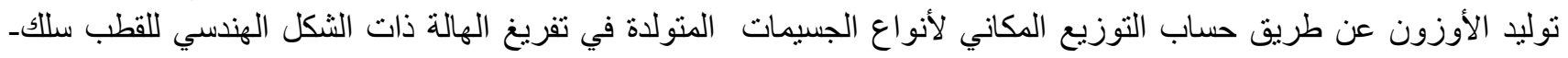

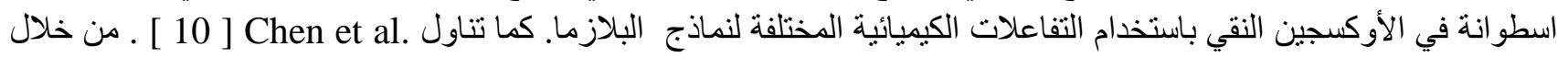

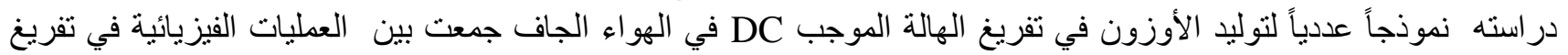

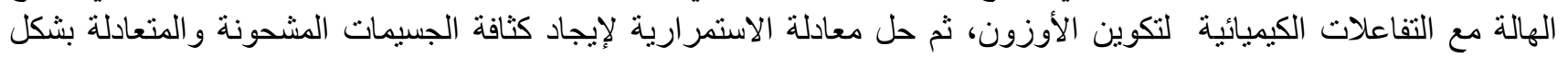

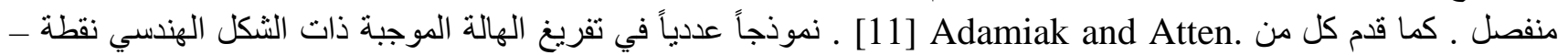
مستوي، إذ نم حساب المجال الكهربائي وكثافة الثحنة من خلال معادلة بوسون ومعادلات الاستمر ارية. 


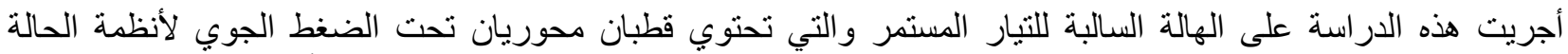

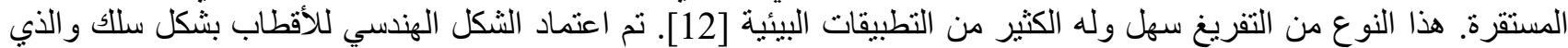

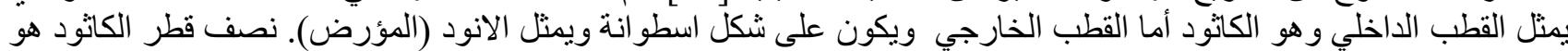

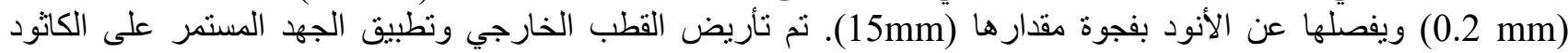

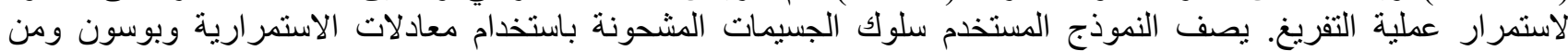

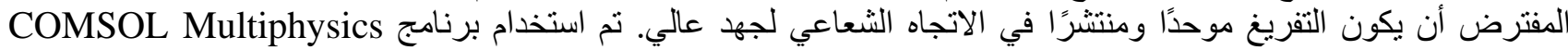

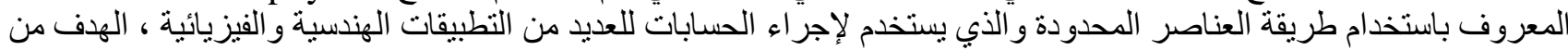

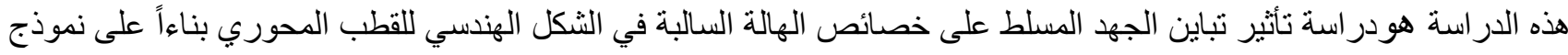
ديناميكي أحادي البعد التفريغ الهالة. معادلات الحاكمة و الاستمر ارية

نموذج النظام الفيزيائي لتفريغ الهالة هو النموذج الهيدروديناميكي يتكون من تدفقات الانجراف والانتشار يمكن تعريف هذا

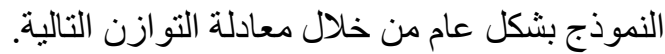

$$
\frac{\partial N}{\partial \mathrm{t}}+\nabla \cdot \Gamma=\mathrm{S}
$$

حيث N و م و S هي كثافة الأنو اع المشحونة والتدفق و مصدر نوليد الإلكترونات على التوالي. يمكن أن يشمل التدفق جزأين

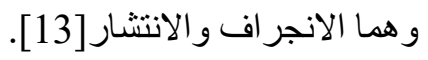

$$
\Gamma=\mu \mathrm{EN}-\mathrm{D} \nabla \mathrm{N}
$$

يمثل الحد الأول الانجر اف ويتناسب مع المجال المسلط E وحركية الأنواع المشحونة بر بينما يمثل الحد الثناني الانتشار ويعتمد على تدرج كثافة الأنو اع ومعامل الانتشار D. ويمكن التعبير عن معامل الانتشار للأيونات الموجبة و السالبة على النئ النحو التالي.

$$
D=\mu k_{B} T_{i} / q
$$

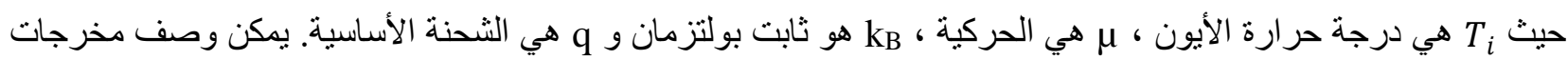

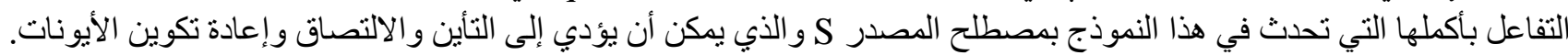
يتم وصف معادلات استمر ارية الجسيمات المشحونة (الأيونات السالبة والأيونات الموجبة و الإلكترونات) في إحداثيات أسطو انية

$\frac{\partial \mathrm{N}_{\mathrm{e}}}{\partial \mathrm{t}}+\frac{1}{\mathrm{r}} \frac{\partial}{\partial \mathrm{r}}\left(\mathrm{rN}_{\mathrm{e}} \mathrm{V}_{\mathrm{e}}\right)-\mathrm{D}_{\mathrm{e}} \frac{1}{\mathrm{r}} \frac{\partial}{\partial \mathrm{r}}\left(\mathrm{r} \frac{\partial \mathrm{N}_{\mathrm{e}}}{\partial \mathrm{r}}\right)=(\alpha-\eta) \mathrm{N}_{\mathrm{e}} \mathrm{V}_{\mathrm{e}}-\beta_{\mathrm{ep}} \mathrm{N}_{\mathrm{e}} \mathrm{N}_{\mathrm{p}}$

$\frac{\partial N_{p}}{\partial t}+\frac{1}{r} \frac{\partial}{\partial r}\left(r N_{p} V_{p}\right)=\alpha N_{e} V_{e}-\beta_{e p} N_{e} N_{p}-\beta_{n p} N_{n} N_{p}$

$\frac{\partial \mathrm{N}_{\mathrm{n}}}{\partial \mathrm{t}}+\frac{1}{\mathrm{r}} \frac{\partial}{\partial \mathrm{r}}\left(\mathrm{rN}_{\mathrm{n}} \mathrm{V}_{\mathrm{n}}\right)=\eta \mathrm{N}_{\mathrm{e}} \mathrm{V}_{\mathrm{e}}-\beta_{\mathrm{np}} \mathrm{N}_{\mathrm{n}} \mathrm{N}_{\mathrm{p}}$

يؤثر نوزيع الهجال الكهربائي اللابلاسي على تجمع الثحنات الفضائية بين الأقطاب الكهربائية ويمكن حسابه بحل معادلة بوسون $\frac{1}{\mathrm{r}} \frac{\partial}{\partial \mathrm{r}}(\mathrm{rE})=-\frac{\mathrm{e}\left(\mathrm{N}_{\mathrm{p}}-\mathrm{N}_{\mathrm{e}}-\mathrm{N}_{\mathrm{n}}\right)}{\varepsilon_{\mathrm{o}}}$

حيث

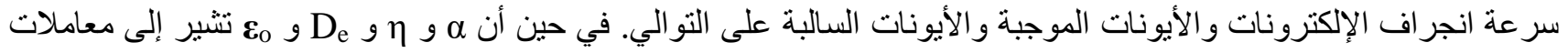

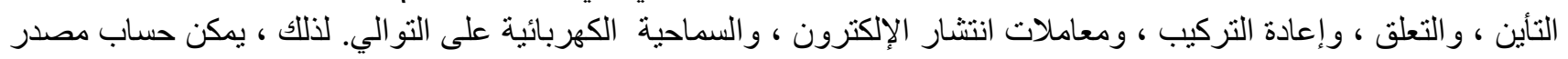

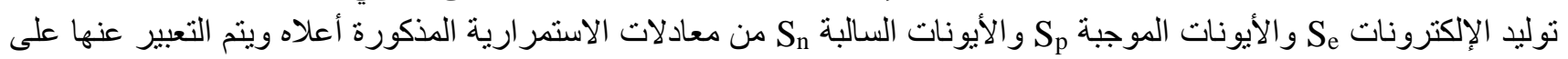
النحو التالي 


$$
\begin{aligned}
& \text { معامل اعادة الاتحاد بين الإلكترونات ـ الأيون الموجب }
\end{aligned}
$$

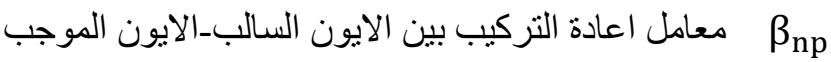

$$
\begin{aligned}
& \mathrm{S}_{\mathrm{e}}=\mathrm{N}_{\mathrm{e}} \mathrm{v}_{\mathrm{e}}-\beta_{\mathrm{ep}} \mathrm{N}_{\mathrm{e}} \mathrm{N}_{\mathrm{p}}(\alpha-\eta) \\
& \mathrm{S}_{\mathrm{p}}=\alpha \mathrm{N}_{\mathrm{e}} \mathrm{v}_{\mathrm{e}}-\beta_{\mathrm{ep}} \mathrm{N}_{\mathrm{e}} \mathrm{N}_{\mathrm{p}}-\beta_{\mathrm{np}} \mathrm{N}_{\mathrm{n}} \mathrm{N}_{\mathrm{p}} \\
& \mathrm{S}_{\mathrm{n}}=\eta \mathrm{N}_{\mathrm{e}} \mathrm{v}_{\mathrm{e}}-\beta_{\mathrm{np}} \mathrm{N}_{\mathrm{n}} \mathrm{N}_{\mathrm{p}}
\end{aligned}
$$

\section{النمذجة العددية}

يمكن تقسيم هذا النموذج أحادي البعد إلى عدد من النقاط مفصولة بمسافة (عناصر المجال) موزعة وفقًا للتسلسل الحسابي

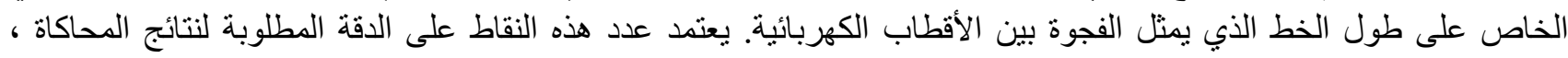

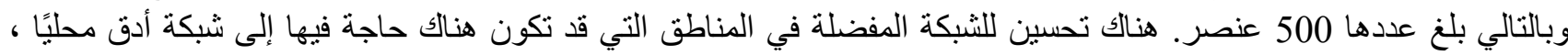

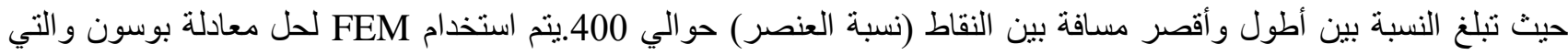

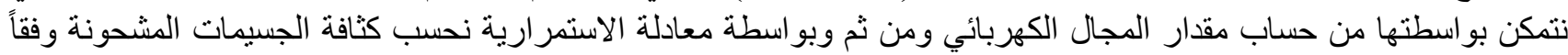

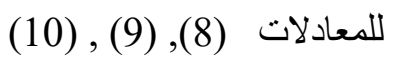

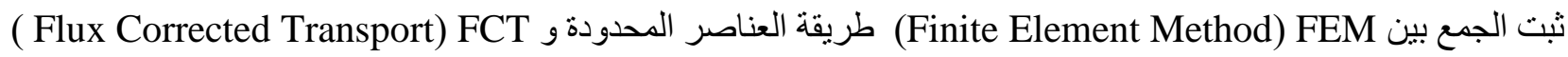

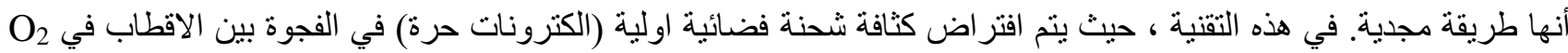

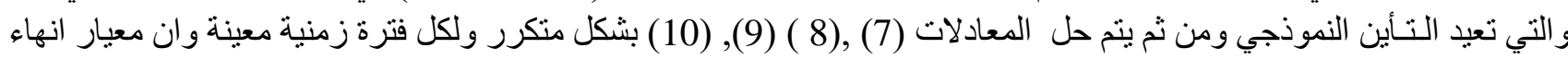

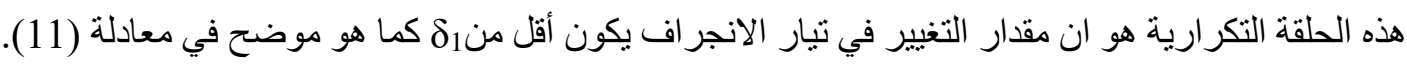

$$
\frac{I_{k+1}-1}{I_{i k+1}} \leq \delta 1
$$

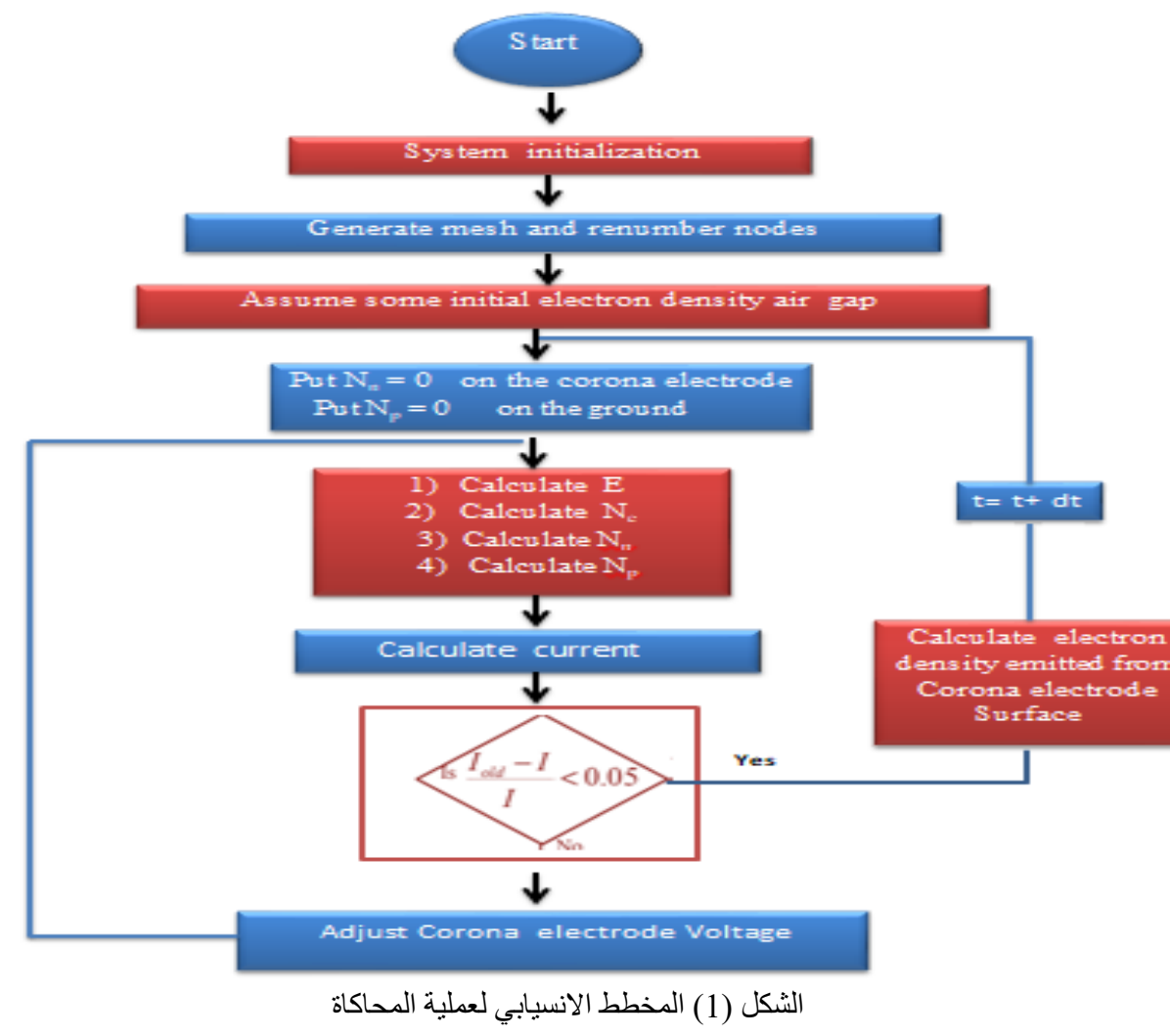


الثرط تم اعتماده للتأكد من تقارب حلول حسابات الفولتية على القطب المسلط عليه الجهد العالي او ما يسمى بقطب الهالة. و هذا

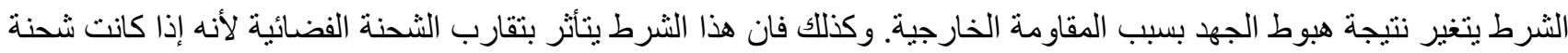

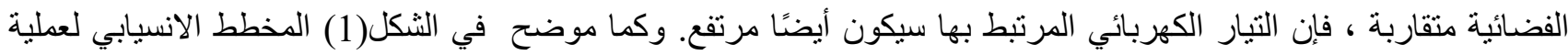

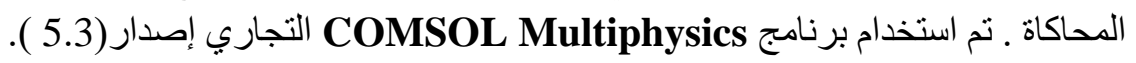

\section{تفاعلات نموذج تفريغ الهالة السالبة في مان}

تم تضمين تسع و عشرون تفاعلًا وأحد عشر نوعًا في النموذج الأولي لتفريغ الهالة السلبي DC في غاز

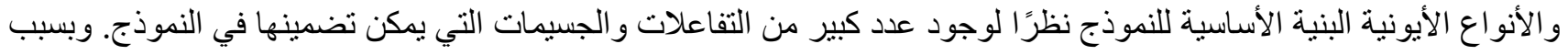

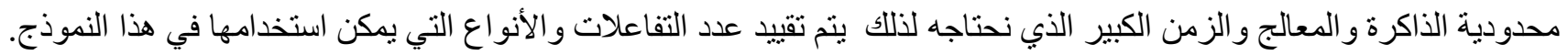

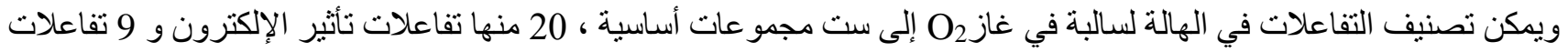

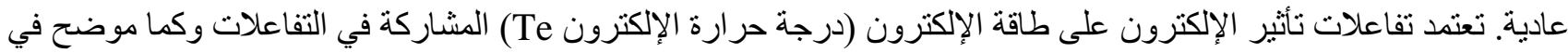

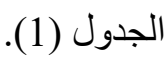

جدول (1) انواع الجسيمات

\begin{tabular}{|ccc|}
\hline Neutral & $\begin{array}{c}\text { Positive charged } \\
\text { species }\end{array}$ & $\begin{array}{c}\text { Negative charged } \\
\text { species }\end{array}$ \\
\hline $\mathrm{O}_{2}$ & $\mathrm{O}_{2}^{+}$ & $\mathrm{O}_{2}^{-}$ \\
$\mathrm{O}_{2} \mathrm{a} 1 \mathrm{~d}$ & & $0-$ \\
$\mathrm{O}_{2} \mathrm{~b} 1 \mathrm{~s}$ & & \\
$\mathrm{O}_{2} 45$ & & \\
0 & & \\
$\mathrm{O}_{1 \mathrm{~d}}$ & \\
$\mathrm{O}_{\mathrm{is}}$ & \\
$\mathrm{O}_{1}$ & \\
\hline
\end{tabular}

تم تضمين أحد عشر نوعًا في جميع التفاعلات ، وثمانية أنواع متعادلة ، ونو عاً موجباً واحداً مشحوناً ونوعين سالبين مشحونين وكلها مدرجة في الجدول (2.2)

يشمل هذا النموذج على تصادمٍ مرنٍ واحدٍ وثلاثة عشر تفاعل تهيج. يتم عرض تفاصيل هذه التفاعلات في الجدول(3.2) يتم

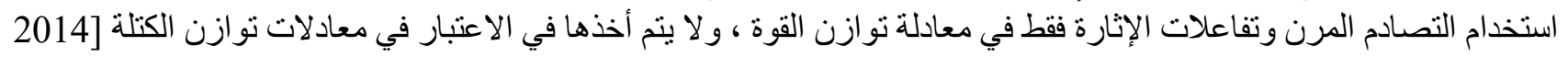

.[Pateau et al , التصادم المرن الذي ينم فيه إعادة توزيع الزخم بين الجسيمات المتصادمة وإجمالي الطاقة الحركية ييقى دون تغيير. ينتج عن مثل

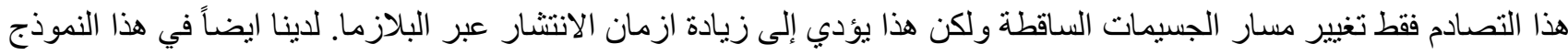

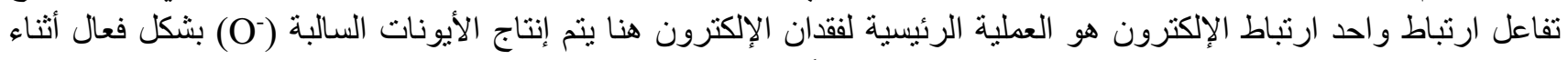

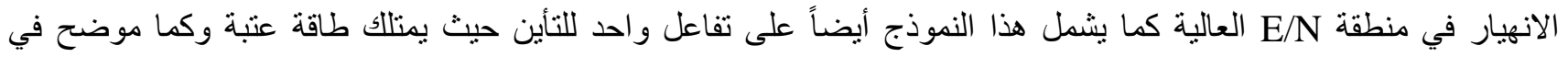

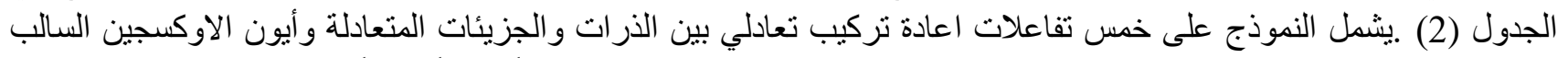

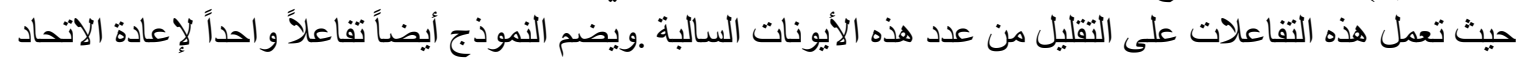


جدول(2) تفاعلات نموذج الهالة السالبة

\begin{tabular}{|c|c|c|c|c|c|}
\hline No. & Reactions & Type & E(ev) & Rate $\left(\mathrm{m}^{3} / \mathrm{s}\right)$ orm $6 / \mathrm{s} \mathrm{.mol}$ & Ref \\
\hline $\mathrm{R}_{1}$ & $\mathrm{e}+\mathrm{O}_{2}=>\mathrm{e}+\mathrm{O}_{2}$ & Elastic scattering & 0.00017 & $f(E)$ & [18] \\
\hline $\mathrm{R}_{2}$ & $\mathrm{e}+\mathrm{O}_{2}=>\mathrm{O}+\mathrm{O}^{-}$ & Attachment & 3.6 & $f(E)$ & [18] \\
\hline $\mathrm{R}_{3}$ & $\mathrm{e}+\mathrm{O}_{2}=>\quad \mathrm{O}_{2}^{-}$ & Attachment & & $2 \times 10^{-41}\left(300 / \mathrm{T}_{\mathrm{c}}\right)$ & [19] \\
\hline $\mathrm{R}_{4}$ & $\mathrm{e}+\mathrm{O}_{2}=>\mathrm{e}+\mathrm{O}_{2}$ & Excitation & 0.02 & $\mathrm{f}(\mathrm{E})$ & [18] \\
\hline $\mathrm{R}_{5}$ & $\mathrm{e}+\mathrm{O}_{2}=>\mathrm{e}+\mathrm{O}_{2}$ & Excitation & 0.193 & $f(E)$ & [18] \\
\hline $\mathrm{R}_{6}$ & $\mathrm{e}+\mathrm{O}_{2}=>\mathrm{e}+\mathrm{O}_{2}$ & Excitation & 0.193 & $f(E)$ & [18] \\
\hline $\mathrm{R}_{7}$ & $\mathrm{e}+\mathrm{O}_{2}=>\mathrm{e}+\mathrm{O}_{2}$ & Excitation & 0.38 & $f(E)$ & [18] \\
\hline $\mathrm{R}_{8}$ & $\mathrm{e}+\mathrm{O}_{2}=>\mathrm{e}+\mathrm{O}_{2}$ & Excitation & 0.38 & $f(E)$ & [18] \\
\hline $\mathrm{R}_{9}$ & $\mathrm{e}+\mathrm{O}_{2}=>\mathrm{e}+\mathrm{O}_{2}$ & Excitation & 0.579 & $f(E)$ & [18] \\
\hline $\mathrm{R}_{10}$ & $\mathrm{e}+\mathrm{O}_{2}=>\mathrm{e}+\mathrm{O}_{2}$ & Excitation & 0.772 & $f(E)$ & [18] \\
\hline $\mathrm{R}_{11}$ & $\mathrm{e}+\mathrm{O}_{2}=>\mathrm{e}+\mathrm{O}_{2} \mathrm{a}_{1} \mathrm{~d}$ & Excitation & 0.977 & $f(E)$ & [18] \\
\hline $\mathrm{R}_{12}$ & $\mathrm{e}+\mathrm{O}_{2}$ ald $=>\mathrm{e}+\mathrm{O}_{2}$ & Disexcitation & 0.977 & $f(E)$ & [18] \\
\hline $\mathrm{R}_{13}$ & $\mathrm{e}+\mathrm{O}_{2} \quad \Rightarrow \mathrm{e}+\mathrm{O}_{2} \mathrm{~b}_{1} \mathrm{~s}$ & Excitation & 1.627 & $f(E)$ & [18] \\
\hline $\mathrm{R}_{14}$ & $\mathrm{e}+\mathrm{O}_{2} \mathrm{~b} 1 \mathrm{~s} \quad \Rightarrow \quad \mathrm{e}+\mathrm{O}_{2}$ & Excitation & 1.627 & $f(E)$ & [18] \\
\hline $\mathrm{R}_{15}$ & $\mathrm{e}+\mathrm{O}_{2} \quad \Rightarrow \quad \mathrm{e}+\mathrm{O}_{2} 45$ & Excitation & 4.5 & $f(E)$ & [18] \\
\hline $\mathrm{R}_{16}$ & $\mathrm{e}+\mathrm{O}_{2} 45 \quad \Rightarrow \quad \mathrm{e}+\mathrm{O}_{2}$ & Disexcitation & 4.5 & $f(E)$ & [18] \\
\hline $\mathrm{R}_{17}$ & $\mathrm{e}+\mathrm{O}_{2} \quad \Rightarrow \mathrm{e}+\mathrm{O}+\mathrm{O}$ & Excitation & 6.1 & $f(E)$ & [18] \\
\hline$\overline{\mathrm{R}_{18}}$ & $\mathrm{e}+\mathrm{O}_{2} \quad \Rightarrow \mathrm{e}+\mathrm{O}+\mathrm{O}_{1} \mathrm{~S}$ & Excitation & 8.4 & $f(E)$ & [18] \\
\hline $\mathrm{R}_{19}$ & $\mathrm{e}+\mathrm{O}_{2} \quad=>\mathrm{e}+\mathrm{O}+\mathrm{O}_{1} \mathrm{~S}$ & Excitation & 9.97 & $f(E)$ & [18] \\
\hline$\overline{\mathrm{R}_{20}}$ & $\mathrm{e}+\mathrm{O}_{2} \Rightarrow>2 \mathrm{e}+\mathrm{O}_{2}^{+}$ & Ionization & & $f(E)$ & [18] \\
\hline $\mathrm{R}_{21}$ & $\mathrm{O}^{-}+\mathrm{O}_{2}=>\quad \mathrm{O}_{3}+\mathrm{e}$ & Recombination & & $3 \times 10^{-16}$ & [20] \\
\hline $\mathrm{R}_{22}$ & $\mathrm{O}^{-}+\mathrm{O}_{2} \quad \Rightarrow \mathrm{O}+\mathrm{O}_{2}+\mathrm{e}$ & Recombination & & $6.9 \times 10^{-16}$ & [20] \\
\hline $\mathrm{R}_{23}$ & $\mathrm{O}^{-}+\mathrm{O}_{3}+\mathrm{e}=>\quad \mathrm{O}_{2}+\mathrm{O}_{2}$ & Recombination & & $1.02 \times 10^{-16}$ & [20] \\
\hline $\mathrm{R}_{24}$ & $+\mathrm{O} \Rightarrow \mathrm{O}_{3}+\mathrm{eO}_{2}^{-}$ & Recombination & & $1.5 \times 10^{-16}$ & [20] \\
\hline $\mathrm{R}_{25}$ & $\mathrm{O}_{2}+\mathrm{O}_{2}+\mathrm{O} \Rightarrow \mathrm{O}_{3}+\mathrm{O}_{2}$ & Association & & $6 \times 10^{-46}$ & [20] \\
\hline $\mathrm{R}_{26}$ & $+\mathrm{O}=>\mathrm{O}_{2}+\mathrm{eO}_{2}^{-}$ & Recombination & & $\mathrm{f}(\mathrm{E})$ & [20] \\
\hline $\mathrm{R}_{27}$ & $\mathrm{O}_{2}+\mathrm{O}_{2}=>\mathrm{O}_{3}+\mathrm{O}$ & Association & & $2.95 \times 10^{-27}$ & [20] \\
\hline $\mathrm{R}_{28}$ & $\mathrm{O}_{2}+\mathrm{O} \Rightarrow>\mathrm{O}_{2}+\mathrm{O}^{-}$ & Ionic exchange & & $1.5 \times 10^{-16}$ & [20] \\
\hline $\mathrm{R}_{29}$ & $\mathrm{O}^{-}+\mathrm{O}_{3} \Rightarrow \mathrm{O}_{2}^{-}+\mathrm{O}_{2}$ & Ionic exchange & & $3.01 \times 10^{-16}$ & [20] \\
\hline
\end{tabular}

\section{النتائج والمناقشة}

يتم محاكاة نموذج تفريغ الهالة لتشمل ثلاثة أنواع مشحونة ( الإكترونات ,الأيونات الموجبة, الأيونات السالبة) حيث يتم من

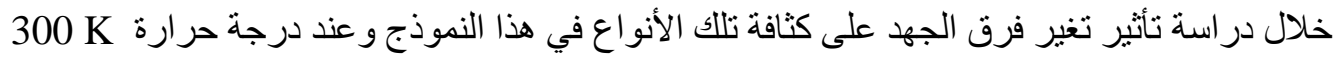
التوزيع الزماني لكثافة التيار الأيوني لقيم مختلفة من فرق الجهد

يبين الثكل (2) العلاقة بين كثافة التيار الأيوني مع تغير زمن الفولتية المسلطة عند خمسة فيم لفروق جهد وهي 7,8,10)KV,

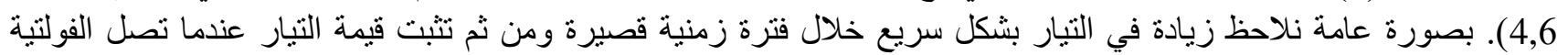

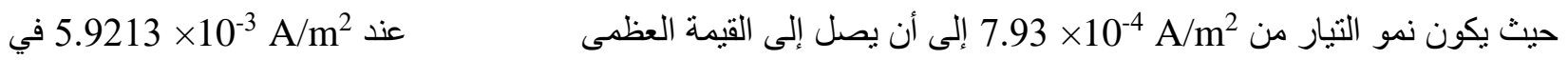

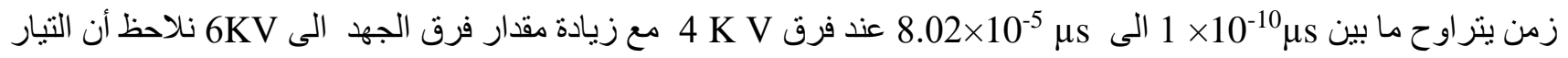


يقلى محافظا على سلوكه مع تغير ملحوظ في قيمته. حيث أن التيار ينمو من 8.8 خلال الزمن ينر اوح بين .4.6110 $\mu^{5} \times s$ s 1 × $10^{-} \mu^{10} \mathrm{~s}$

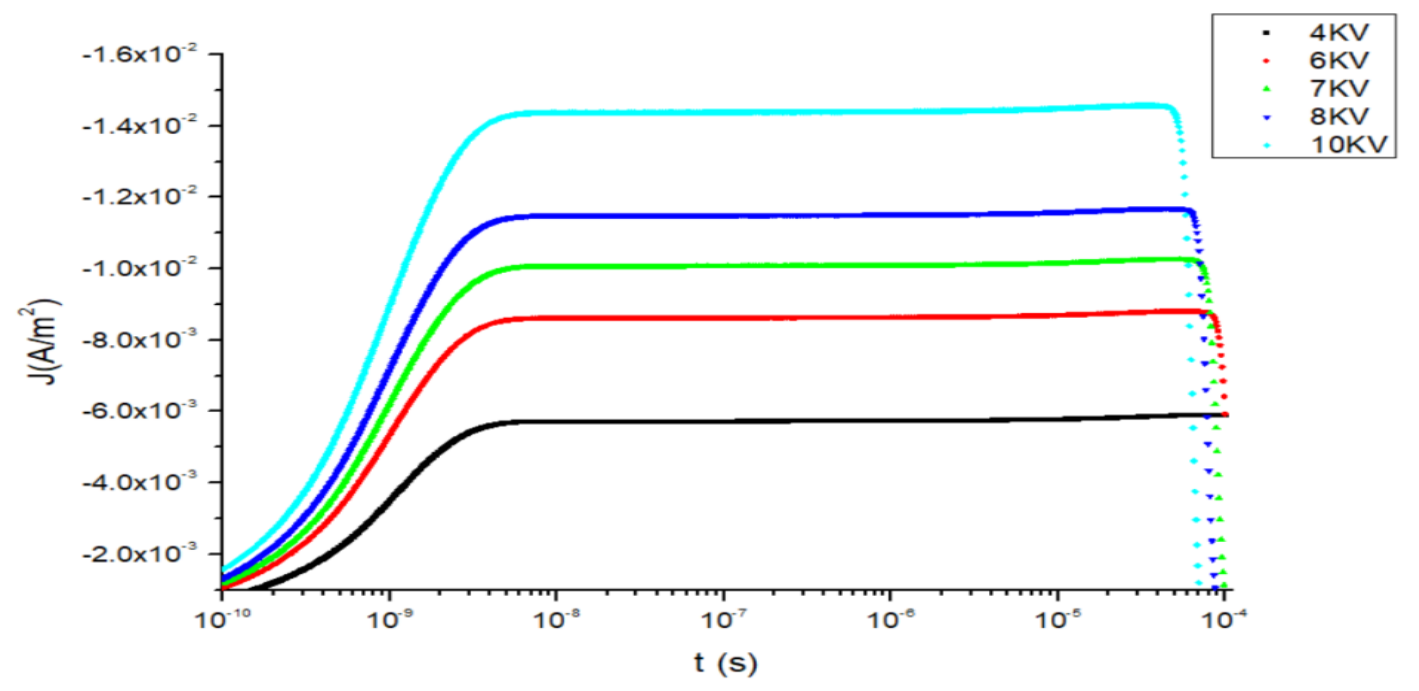

الثكل (2) تغير كثافة التيار الايوني مع الزمن عند تغير فرق الجها

عندما يصل فرق الجهد إلى 7KV نجد أن التيار يبقى محافظاً على سلوكه مع تغير واضح في قيمته، إذ يبدأ التيار بالنمو من

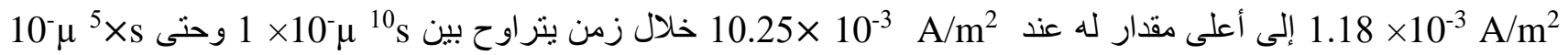

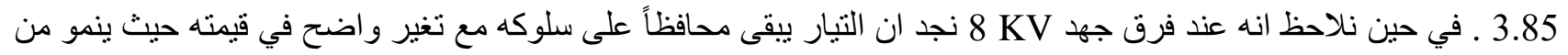

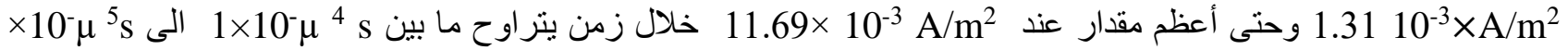

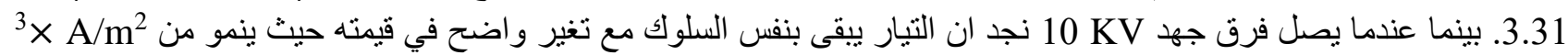

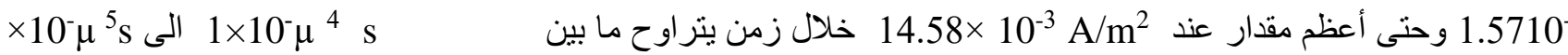

نلاحظ مما سبق ان القيمة العظمى لكثافة التيار ترتفع نحو الاعلى مع زيادة فرق الجهد المسلط ويعزى سبب ذلك انه عند بزيادة

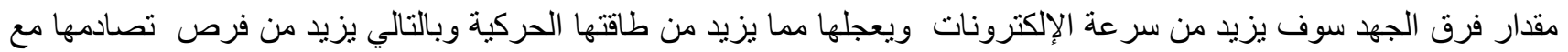

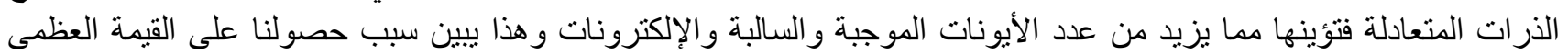

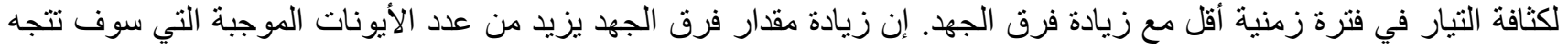

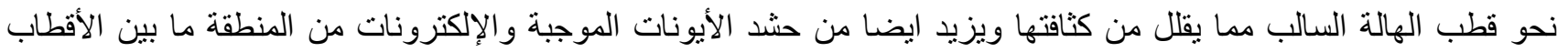

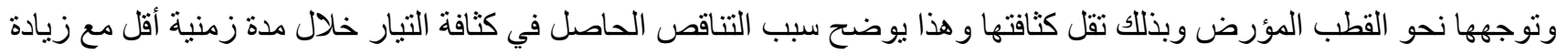
فرق الجهد. التوزيع المكاني لكثافة الإلكترون عند فيم مختلفة من فرق الجهر يبين الثكل 3a نوزيع كثافة الإلكترونات مكانياً لقيم مختلفة من فرق الجهد عند زمن أعلى قيمة للتيار، إذ نلاحظ من خلال الثكل أن كثافة الإكترونات تثناقص عند زيادة فرق الجهد، في حين تبلغ كثافة الإلكترونات

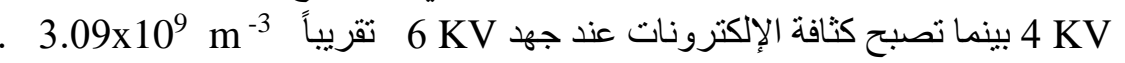

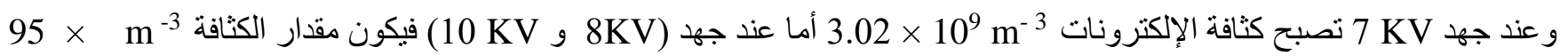
من $2.10^{9}$ من القطب المؤرض حيث تبلغ كثافتها عند

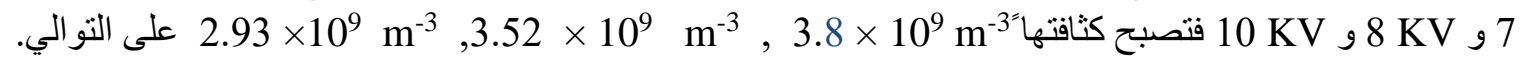


السبب في ذلك يعود لتفاعلات الارتباط حيث أنه بزيادة فرق الجهد تتعجل الإلكترونات ونزداد طاقتها الحركية بحيث أنها تمتلك الكي طاقة كافية لتفكيك جزيئة الاوكسجين عند تتصادم معهاوينتج عن ذلك إلكترون مع ذرني اوكسجين وحسب الإن التفاعل الآتي :

$$
\mathrm{e}+\mathrm{O}_{2}=>\mathrm{O}+\mathrm{O}+\mathrm{e}
$$

الإلكترونات الناتجة التي تمنلك طاقة قليلة يمكنها الارتباط بإحدى ذرتي الاوكسجين الناتجة ولهذا تقل كثافة الالكترونات بزيادة فرق

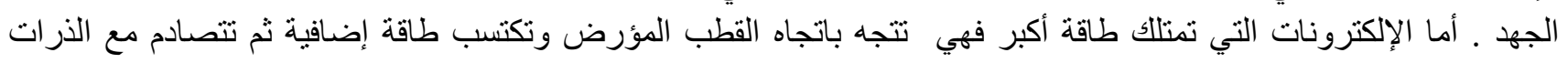

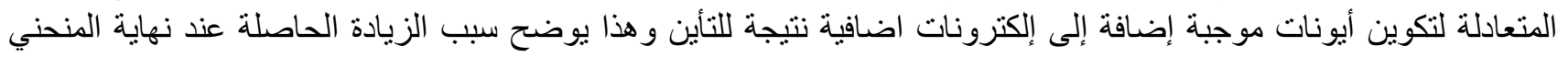

وكما موضح في الثكل (3b).

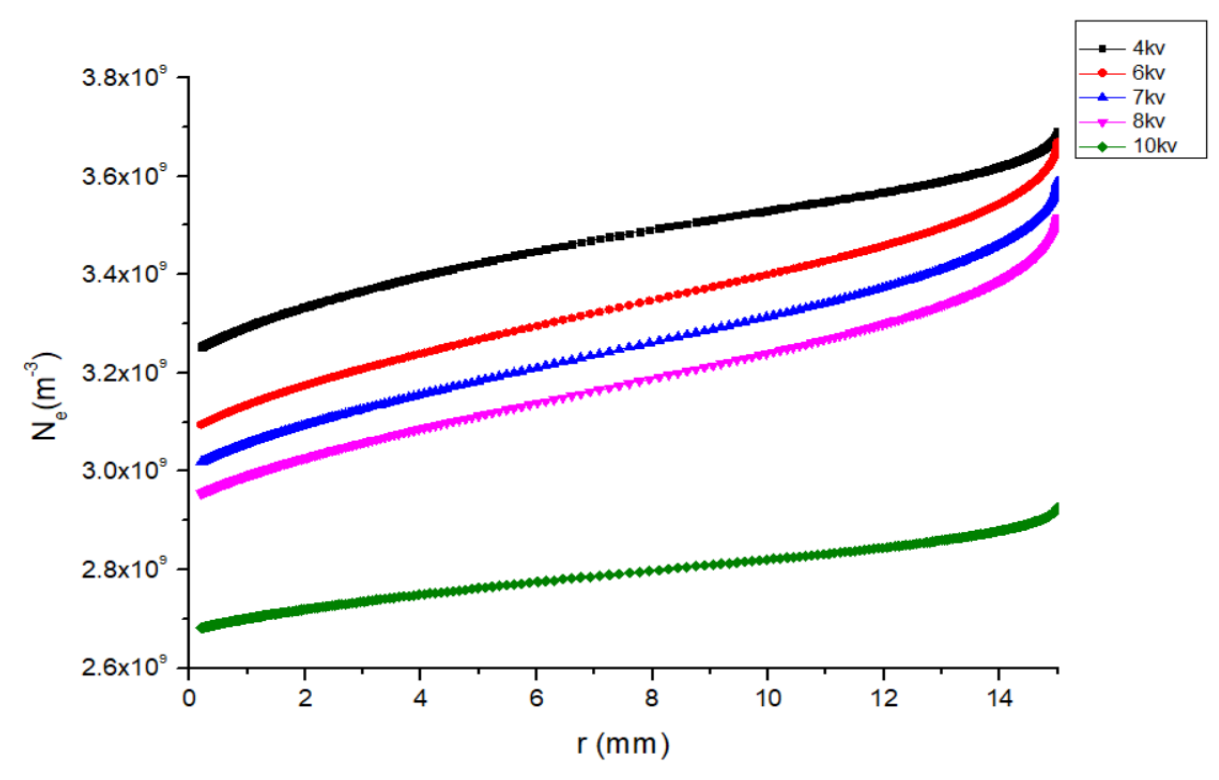

(a)

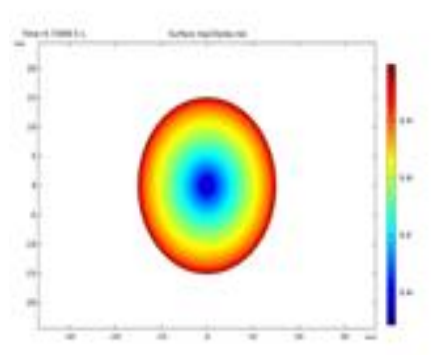

$7 \mathrm{kV}$

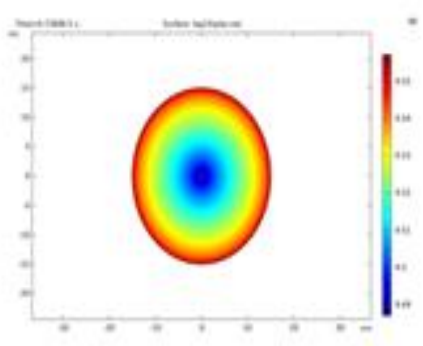

$6 \mathrm{kV}$

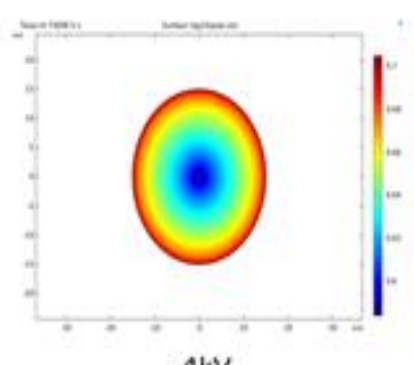

$4 \mathrm{kV}$

(b)

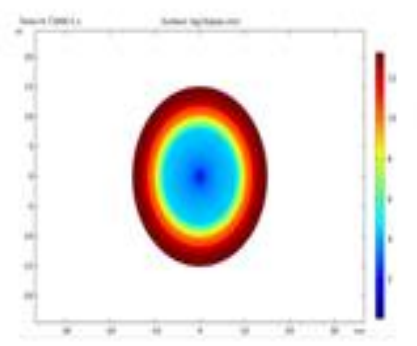

$10 \mathrm{kV}$

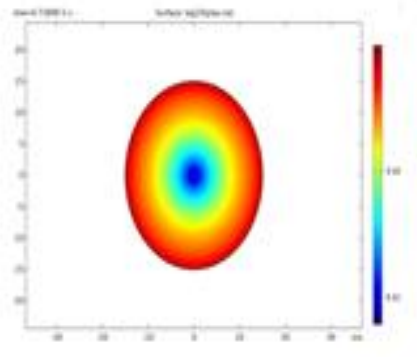

$8 \mathrm{kV}$

الثكل (3) a التوزيع المكاني لكثافة الإكترونات لقيم مختلفة من فرق الجها عند زمن أعلى قيمة للتيار الثكل (3) b. التوزيع السطحي ثنائي الأبعاد لكثافة الإكترون لتفريغ الهالة السالب لقيم مختلفة لفرق الجها 
التوزيع المكاني لكثافة الأيونات الموجبة لقيم مختلفة من فرق الجهد

يبين الثكل (4a) توزيع كثافة الأيونات الموجبة مكانياً مع قيم مختلفة لفرق الجهد المسلط عند زمن أعلى قيمة للتيار. نلاحظ

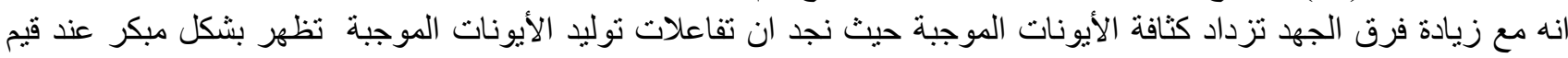

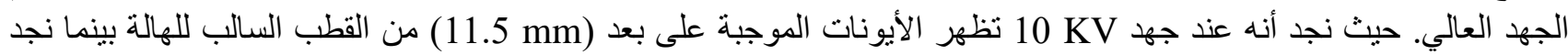

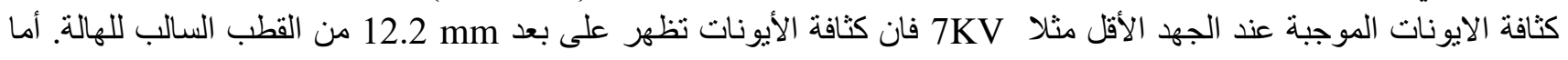

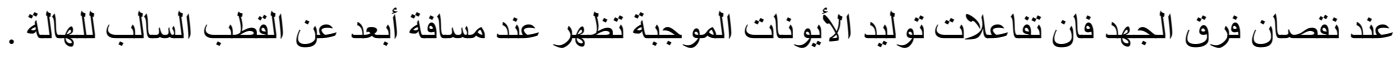

لتفسير ذلك أنه عند الجهد العالي فإن الإكترونات تكتسب طاقة كافية من فرق الجهد لمثل هكذا تفاعلات فتتصادم مع الذرات

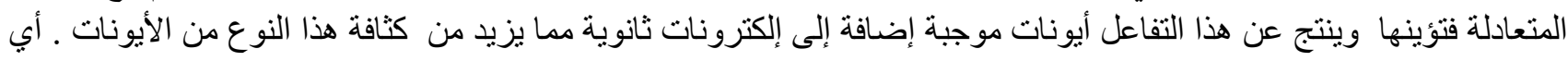

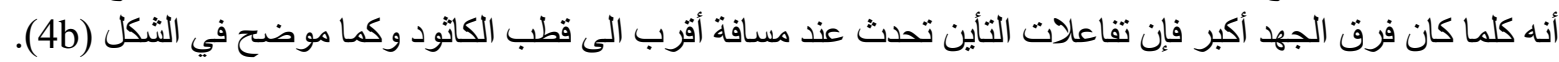



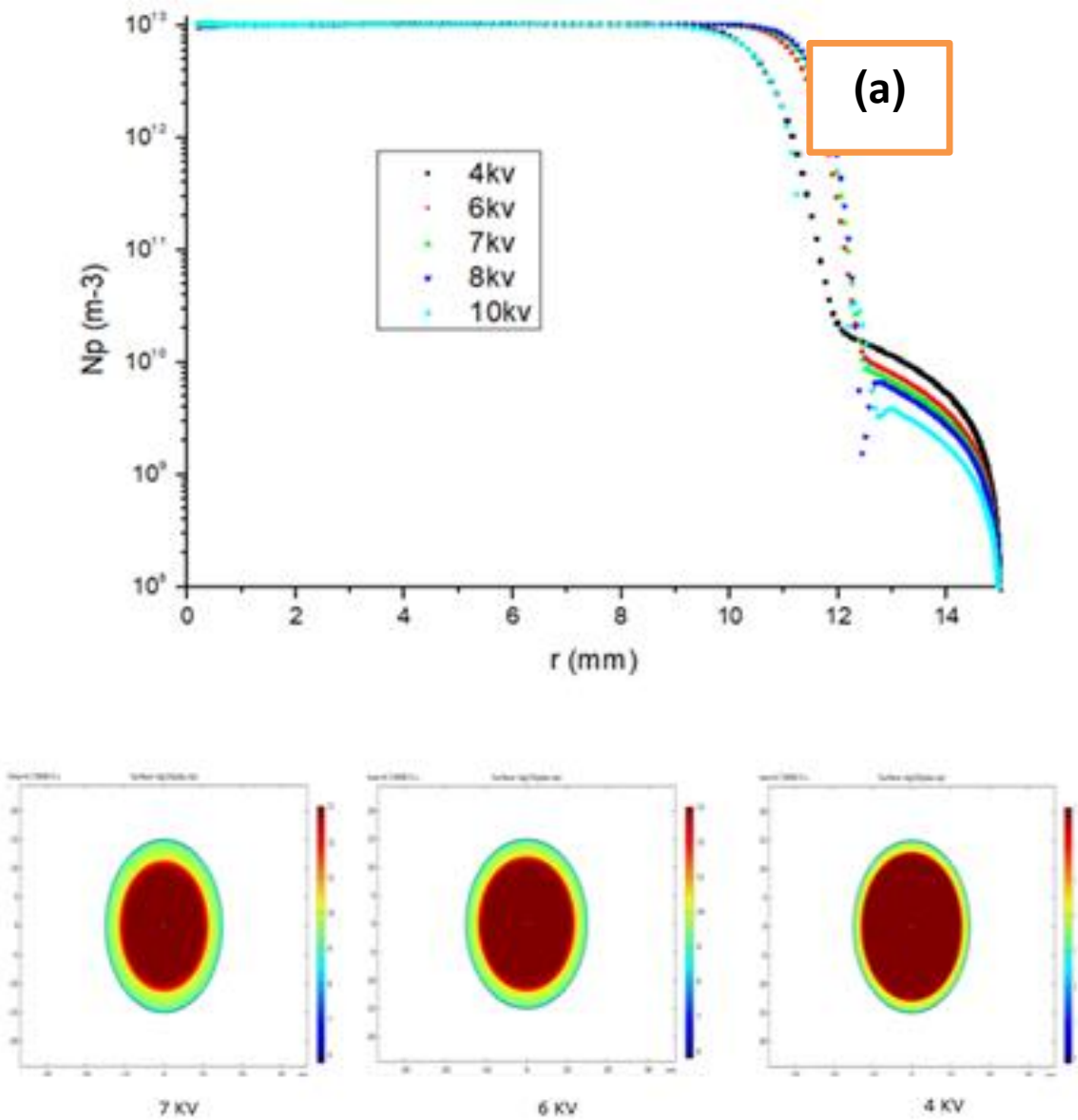

$(4)$
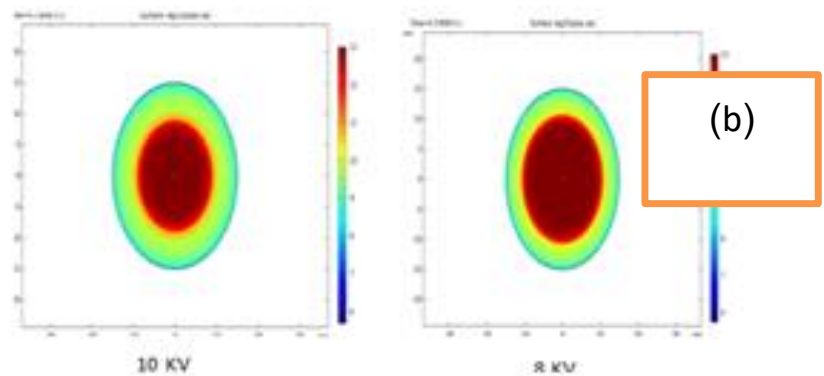

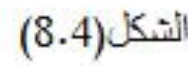

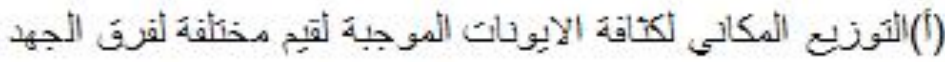

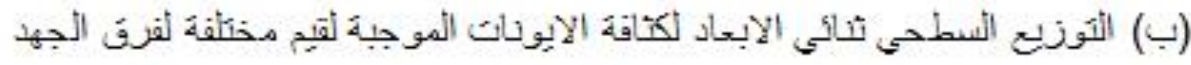

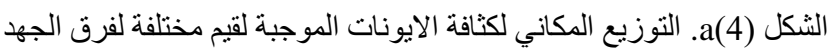

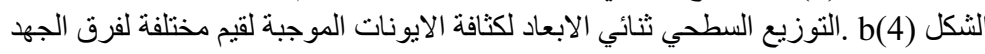


التوزيع المكاني لكثافة الأيونات السالبة لقيم مختلفة لفرق الجهد

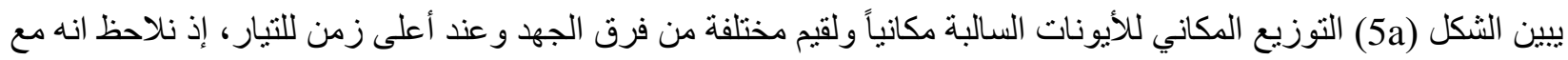

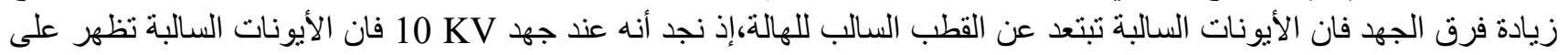

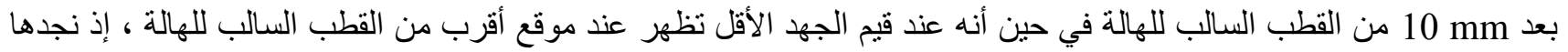

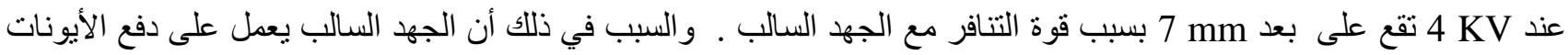

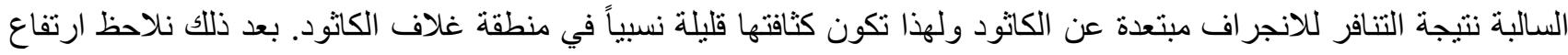

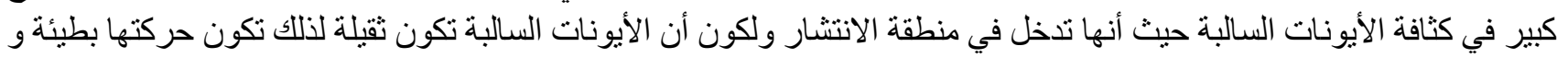

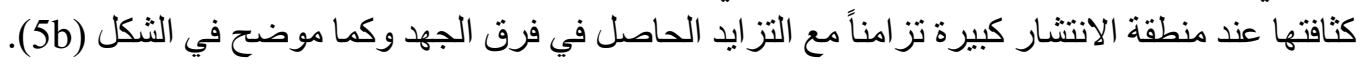
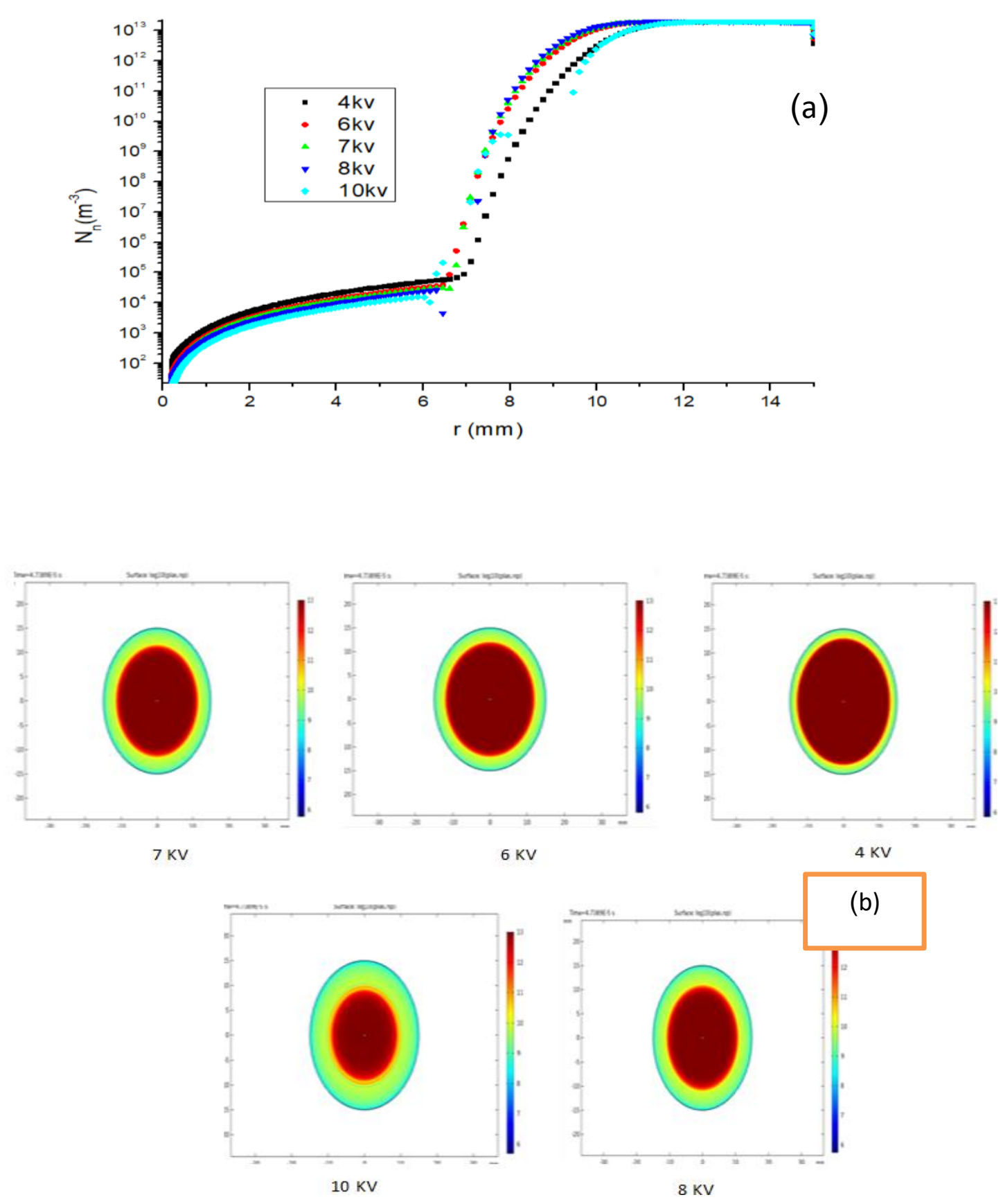

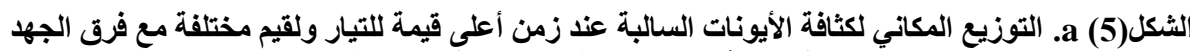

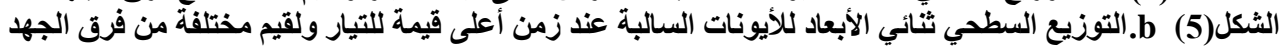


تغير كثافة الاوزون مع فرق الجهذ

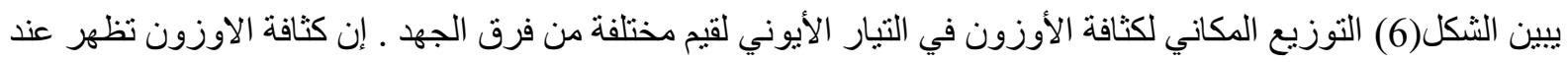

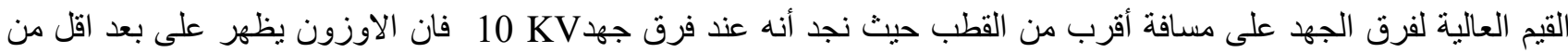

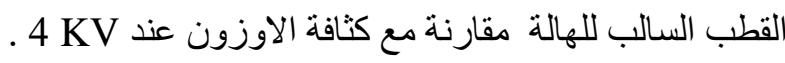

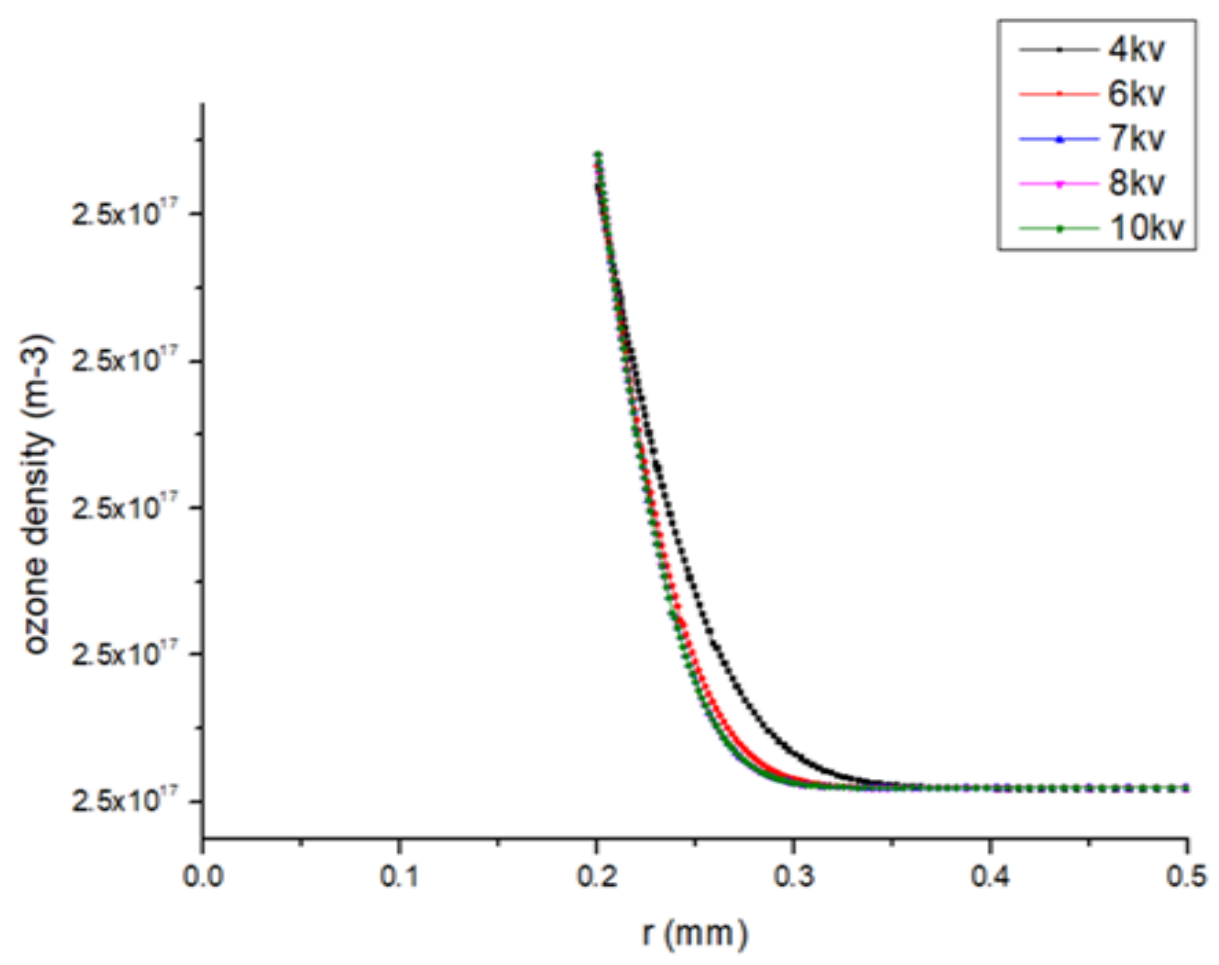

وسبب ذلك يعود إلى أنه عند زيادة فرق الجهد سوف يمتلك الإلكترون طاقة كافية لعملية تفكك الاوكسجين ومن ثم تتفاعل هذه الذرة مع جزيئة اوكسجين لتكوين الاوزون وحسب الني التفاعل الآتي

$$
\mathrm{e}+\mathrm{O}_{2}=>2 \mathrm{O}+\mathrm{e}
$$$$
\mathrm{O}_{2}+\mathrm{O}+\mathrm{M}=>\mathrm{O}_{3}+\mathrm{M}
$$

يحدث التفكك عند الجهد العالي في نقطة أقرب الى القطب منه عند الجهد الأقل وذلك بسبب أن تأثير الجهد الخارجي على توليد

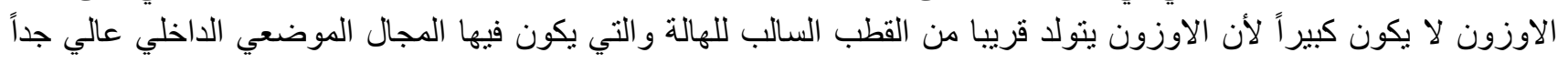

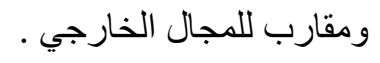
الاستتناجات

القيمة العظمى لكثافة التيار ترتفع نحو الأعلى مع زيادة فرق الجهد المسلط. في حين تتخفض كثافة التيار الأيوني في فترة زمنية

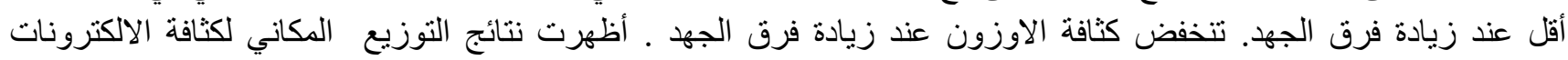

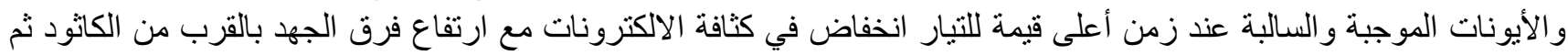

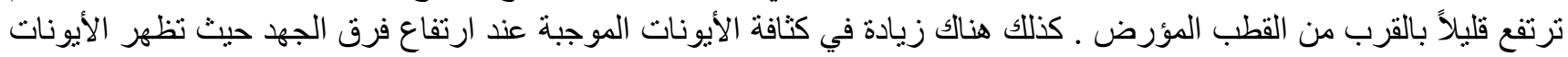

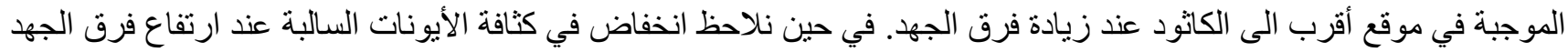

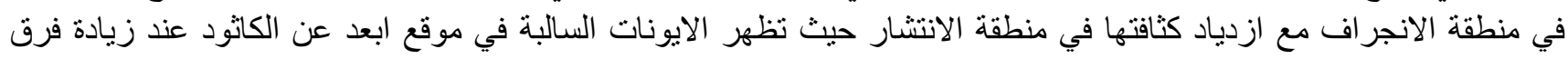




\section{Acknowledgments}

Special thankfulness and gratefulness go to the University of Mosul and the College of Education for Pure Science for their cooperation to carry out this research and also to give me great support and opportunity to study Master in Physics.

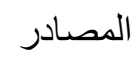

1. Li, Z.X.; Li, G.F.; Fan, J.B. and Yin, Y., European Transactions on Electrical, 21:11-17 (2011).

2. Oglesby, S. and Nichols, G.B. "Electrostatic precipitation." Marcel Dekker, New York (1978).

3. Holboell, J. and Sverrisson, S., 24th Nordic Insulation Symposium, 24:134-137 (2015).

4. Arora, R. and Mosch, W., 'High Voltage and Electrical Insulation Engineering'. Wiley, Hoboken, NJ, USA. (2011).

5. Jassim, M.K.; Jawad, E.A. and Alsaide, J.K. "Effect of temperature on the working parameters of negative corona discharge with coaxial electrodes configuration." Iraqi Journal of Science.:

6. Basirabad, P.D., Electronic Thesis and Dissertation Repository, P 4167 (2016).

7. Tran, T. N.; Golosnoy, I. O.; Lewin P. L. and Georghiou', G. E." Numerical modelling of negative discharges in air with experimental validation" Journal of Physics D: Applied Physics, IOP, 44 (1): 15203 (2011).

8. Panicker, P. K. M.Sc. Thesis, Faculty of the Graduate School, University of Texas (2003).

9. Pontiga, F.; Soria, C.; Castellanos, A; Skanly, J.D. An study of ozone generation by negative corona discharge through different plasma chemistry models, Ozone Science and Engineering 24: 447-462. (2002).

10. Chen, J.H; Davidson, J.H. Electron density and energy distributions in the positive DC corona: interpretation for corona-enhanced chemical reactions, Plasma Chemistry and Plasma Processing 22: 199-224 (2002) .

11.Adamiak, K. ; Atten, P. Simulation of corona discharge in pointeplane configuration, Journal of Electrostatics 61: 85-98 (2004)

12. Veldhuizen, E. M. 'Electrical Discharges for Environmental Purposes: Fundamentals and Applications', Nova Science Publishers, New York (2000).

13. Karman, G., M.Sc. Thesis, Division of High Voltage Engineering, Chalmers University of Technology (2013).

14. Chen, J. and Davidson, J.H., Plasma Chem. Plasma Process, 23(1): 83-102 (2003).

15. Kim, N.; Lee, S.; Georghiou, G.E. and Kim, D., J. Electr. Eng. Tech., 7(1):97-102 (2012).

16.Yanallah, K.; Pontiga, F. and Castellanos, A., J. Phys. D: Appl. Phys., 44(5):55201 (2011).

17. Saleh, Dawood N.; Algwari, Qais Th. And Amouri, Farook Kh. Modeling the dependence of the negative corona current density on applied voltage rise time : Phys. Plasmas 27, 073501 (2020).

18.BOLSIG+, Electron Boltzmann equation solver. https://www. bolsig.laplace.univ-tlse.fr.

19. Liu, K.L.; Liao, R.J. and Zhao, X.T., J. Electr. Eng. Technol., 10(4):1804-1814 (2015).

20. LXCAT, "Electron scattering database." University of Toulouse, France. www.lxcat.net. (2017). 